\title{
Vascular thrombus imaging in vivo via near-infrared fluorescent nanodiamond particles bioengineered with the disintegrin bitistatin (Part II)
}

This article was published in the following Dove Press journal:
International Journal of Nanomedicine

Number of times this article has been viewed

\author{
Jonathan A Gerstenhaber ${ }^{1, *}$ \\ Frank C Barone ${ }^{2, *}$ \\ Cezary Marcinkiewicz ${ }^{1,3}$ \\ Jie $\mathrm{Li}^{2}$ \\ Aaron O Shiloh ${ }^{4}$ \\ Mark Sternberg ${ }^{3}$ \\ Peter I Lelkes ${ }^{1, *}$ \\ Giora Feuerstein ${ }^{1,3, *}$ \\ 'Department of Bioengineering, \\ College of Engineering, Temple \\ University, Philadelphia, PA, \\ ${ }^{2}$ Department of Neurology, State \\ University of New York Downstate \\ Medical Center, Brooklyn, NY, ${ }^{3}$ Debina \\ Diagnostic Inc., Newtown Square, \\ ${ }^{4}$ Diagnostic Imaging, Inc., Philadelphia, \\ PA, USA \\ *These authors contributed equally \\ to this work
}

\begin{abstract}
The aim of this feasibility study was to test the ability of fluorescent nanodiamond particles (F-NDP) covalently conjugated with bitistatin (F-NDP-Bit) to detect vascular blood clots in vivo using extracorporeal near-infrared (NIR) imaging. Specifically, we compared NIR fluorescence properties of F-NDP with N-V $\left(\mathrm{F}_{-N D P}\right)$ and N-V-N color centers and sizes (100-10,000 nm). Optimal NIR fluorescence and tissue penetration across biological tissues (rat skin, porcine axillary veins, and skin) was obtained for $\mathrm{F}_{-\mathrm{NDP}}$ with a mean diameter of $700 \mathrm{~nm}$. Intravital imaging (using in vivo imaging system [IVIS]) in vitro revealed that F-NDP ${ }_{\mathrm{NV}}$-loaded glass capillaries could be detected across $6 \mathrm{~mm}$ of rat red-muscle barrier and $12 \mathrm{~mm}$ of porcine skin, which equals the average vertical distance of a human carotid artery bifurcation from the surface of the adjacent skin $(14 \mathrm{~mm})$. In vivo, feasibility was demonstrated in a rat model of occlusive blood clots generated using $\mathrm{FeCl}_{3}$ in the carotid artery bifurcation. Following systemic infusions of F-NDP ${ }_{\mathrm{NV}}$-Bit (3 or $15 \mathrm{mg} / \mathrm{kg}$ ) via the external carotid artery or femoral vein $(\mathrm{N}=3)$, presence of the particles in the thrombi was confirmed both in situ via IVIS, and ex vivo via confocal imaging. The presence of $\mathrm{F}_{-\mathrm{NDP}} \mathrm{NV}_{\mathrm{N}}$ in the vascular clots was further confirmed by direct counting of fluorescent particles extracted from clots following tissue solubilization. Our data suggest that $\mathrm{F}-\mathrm{NDP}_{\mathrm{NV}}-\mathrm{Bit}$ associate with vascular blood clots, presumably by binding of $\mathrm{F}-\mathrm{NDP}_{\mathrm{NV}}$-Bit to activated platelets within the blood clot. We posit that $\mathrm{F}-\mathrm{NDP}{ }_{\mathrm{NV}}$-Bit could serve as a noninvasive platform for identification of vascular thrombi using NIR energy monitored by an extracorporeal device.
\end{abstract}

Keywords: fluorescent nanodiamond particles, NIR fluorescence imaging, thrombosis, biomarkers

\section{Introduction}

Thromboembolic events (TEE) in cerebral and coronary vessels are the major causes of death from strokes and heart attacks in developed countries. ${ }^{1,2}$ Advanced technologies such as computed tomography (CT) scans, magnetic resonance imaging (MRI), and angiography are routinely used to identify the location of tissue ischemia or infarct and guide treatment options in conditions such as focal occlusive stroke and heart attacks. ${ }^{3}$ However, these diagnostic technologies have drawbacks that preclude their routine utilization for primary disease risk assessment in an ambulatory setting. For example, MRI requires specialized facilities and specialty-trained medical teams, and is restrictive in terms of costs and availability in non-tertiary (rural, community) medical facilities. CT scans, especially angiography, require hospital-based facilities and highly trained technical and medical personnel to acquire and interpret the complex imaging information. Furthermore, most current imaging technologies used for assessing TEE are
Correspondence: Cezary Marcinkiewicz Department of Bioengineering, College of Engineering, Temple University, 1947 North I2th Street, Philadelphia, PA 19122, USA

Tel +I 2152043307

Email cmarcink@temple.edu 
indirect; that is, they monitor morphological changes, such as the narrowing of blood flow, rather than pinpointing vascular lesion content (eg, presence of a blood clot vs atherosclerotic lesion). Ultrasound imaging of vascular elements provides mostly dimensional information precluding assessment of specific pathological elements such as blood clots associated with a vulnerable plaque. Altogether, the ability to evaluate the risk of TEE as part of the general health management for primary prevention of vascular thrombosis remains a significant unmet medical need. These considerations suggest the need for advanced technology to provide specific knowledge of "vascular pathology content", which could be acquired noninvasively in real time and in an affordable ambulatory setting.

We recently embarked on the bioengineering of fluorescent nanodiamond particles (F-NDP) covalently coupled to the disintegrin protein bitistatin (F-NDP-Bit) for the detection of vascular blood clots via specific binding of the combined particle (F-NDP-Bit with N-V-N color centers, F-NDP ${ }_{\mathrm{NVN}^{-}}$ Bit) to the activated platelet fibrinogen receptor. ${ }^{4} \mathrm{~F}-\mathrm{NDP}$ as a platform are capable of diverse functionalization and have been coupled to antibodies for protein detection, adsorbed with small molecules for therapeutic purposes, and even inorganically doped for use as catalysts..$^{5-7}$ Our previous set of in vitro studies, in which we demonstrated the specific association of the F-NDP ${ }_{\mathrm{NVN}}$-Bit with platelets embedded within plasma clot generated by thrombin-activated rat platelet-rich plasma, provided a first-line "proof of feasibility" for our concept. ${ }^{4}$ Since transmission of light across biological tissues is subject to interferences (scatter, absorption, diffraction) by organic and inorganic materials of the tissues, ${ }^{8}$ we have primarily focused on the near-infrared (NIR) optical window, which significantly facilitates penetration of light through biological tissues. ${ }^{8-10}$ However, F-NDP with N-V-N color centers $\left(\mathrm{F}-\mathrm{NDP}_{\mathrm{NVN}}\right)$ used in our prior study deliver limited energy in the NIR window, and will most likely fail to penetrate over physiologically relevant distances that could serve medical objectives. Therefore, we expanded our search for an F-NDP strain more suitable to deliver the energy needed to survey vascular lesions such as vascular blood clots. We now report on comprehensive in vitro, in vivo, and in situ studies conducted with F-NDP-Bit with N-V color centers (F-NDP ${ }_{\mathrm{NV}}$-Bit) that emit strong NIR fluorescence necessary for deep imaging of blood clot in vascular tissue of the human body via an extracorporeal device.

\section{Materials and methods}

\section{Materials}

Bitistatin was purified from the venom of Bitis arietans (Latoxan Serpentarium, Valence, France) using two steps of reverse-phase high-performance liquid chromatography, as described previously. ${ }^{4}$ F-NDP, chemically surfacefunctionalized with carboxyl groups $(-\mathrm{COOH})$, were purchased from Adamas Nanotechnologies (Raleigh, NC, USA). Two strains of F-NDP were used: green fluorescent F-NDP $_{\text {NVN }}$ at $700 \mathrm{~nm}\left(2 \times 10^{8}\right.$ particles $\left./ \mathrm{mg}\right)$ and red fluorescent F-NDP with N-V color centers $\left(F-N_{N V}\right)$ at $100 \mathrm{~nm}$ $\left(5 \times 10^{11}\right.$ particles/mg $), 700 \mathrm{~nm}\left(2 \times 10^{8}\right.$ particles $\left./ \mathrm{mg}\right)$, and $10,000 \mathrm{~nm}\left(5 \times 10^{5}\right.$ particles/mg). Isoflurane (IF; B34C16A) was purchased from Henry Schein (Melville, NY, USA). Ethyl alcohol (70\% denatured) and PE-10 tubing were purchased from Thermo Fisher Scientific (Waltham, MA, USA). Roboz SUT-15-1 5-0 silk suture was purchased from Roboz Surgical Instrument Co. (Gaithersburg, MD, USA). Parafilm and $\mathrm{FeCl}_{3}$ were purchased from Sigma-Aldrich (St Louis, MO, USA).

\section{Coupling of bitistatin to F-NDP}

Bitistatin was coupled to the F-NDP of all types using 1-ethyl-3-[3-dimethylaminopropyl] carbodiimide hydrochloride (EDC) as a hetero-bifunctional cross-linker, ${ }^{11}$ according to the methodology described previously. ${ }^{4}$ Coupling efficiency and preservation of bitistatin activity on the various functionalized nanodiamond particles (F-NDP-Bit) were verified using a semiquantitative enzyme-linked immunosorbent assay, as described previously. ${ }^{4}$

\section{Characterization of NIR emission of $\mathrm{F}_{-N D P_{\mathrm{NV}}}$ and $\mathrm{F}-\mathrm{NDP}_{\mathrm{NVN}}$}

NIR fluorescence profiles of F-NDP were characterized using a Tecan Infinite 200 PRO (Tecan AG, Männedorf, Switzerland). One hundred microliters of $3 \mathrm{mg} / \mathrm{mL}$ of $700 \mathrm{~nm} \mathrm{F-NDP}{ }_{\mathrm{NV}}$ or F-NDP ${ }_{\mathrm{NVN}}$ suspended in de-ionized (DI) water was loaded into 96-well polystyrene. Fluorescence was scanned for all wells with excitations from 230 to $850 \mathrm{~nm}$ and emissions from 290 to $850 \mathrm{~nm}$ (Figure 1A) at $20 \mathrm{~nm}$ intervals. Data were processed in MATLAB 2015b (Mathworks, Natick, MA, USA). Background fluorescence was subtracted from empty wells without F-NDP, and the resulting net fluorescence value was $\log _{10}$-transformed for visualization.

Glass capillaries (40 mm length, $1 \mathrm{~mm}$ internal diameter; Thermo Fisher Scientific) were filled with equal volumes $(30 \mu \mathrm{L})$ of suspensions of F-NDP at concentrations from $0.06 \mathrm{mg} / \mathrm{mL}$ and up to $4 \mathrm{mg} / \mathrm{mL}(1.8-120 \mu \mathrm{g}$ total particle mass) and sealed at each end by plasticine (Hasbro, Pawtucket, RI, USA). The NIR fluorescence intensity of the various suspensions in the capillaries was analyzed using an in vivo imaging system (IVIS; IVIS 50 Imaging System; 

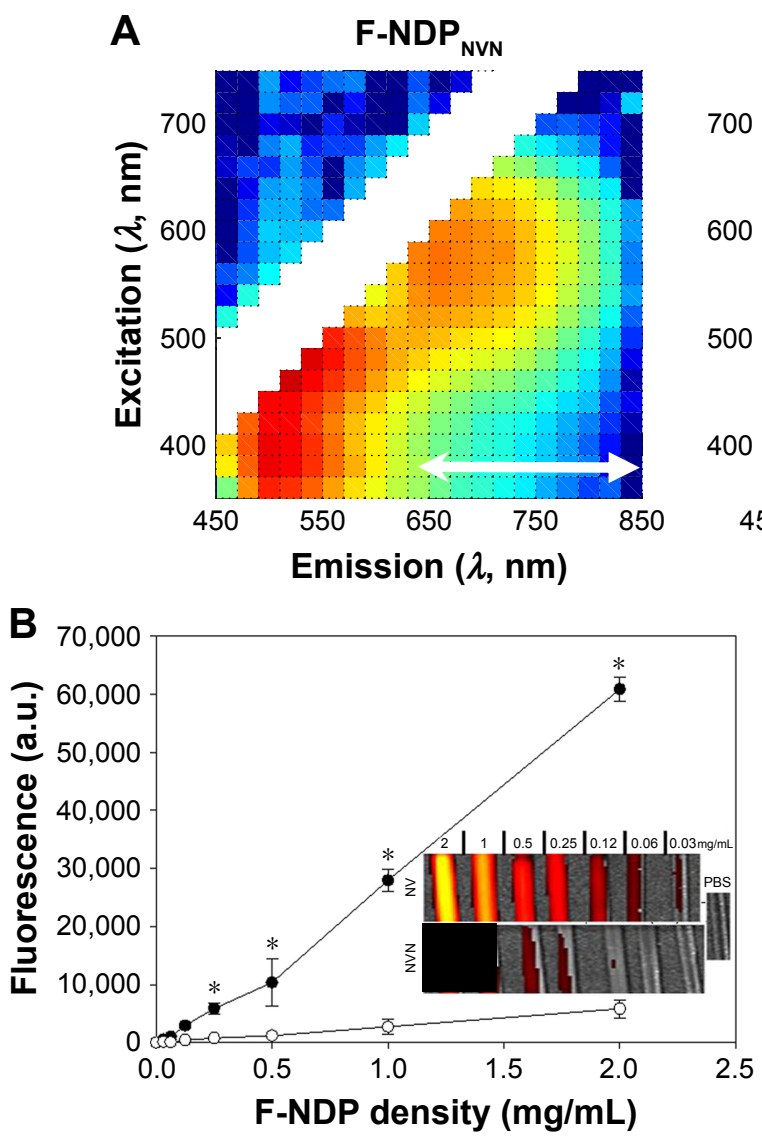

$\bullet N V($ red) $\multimap-N V N$ (green)
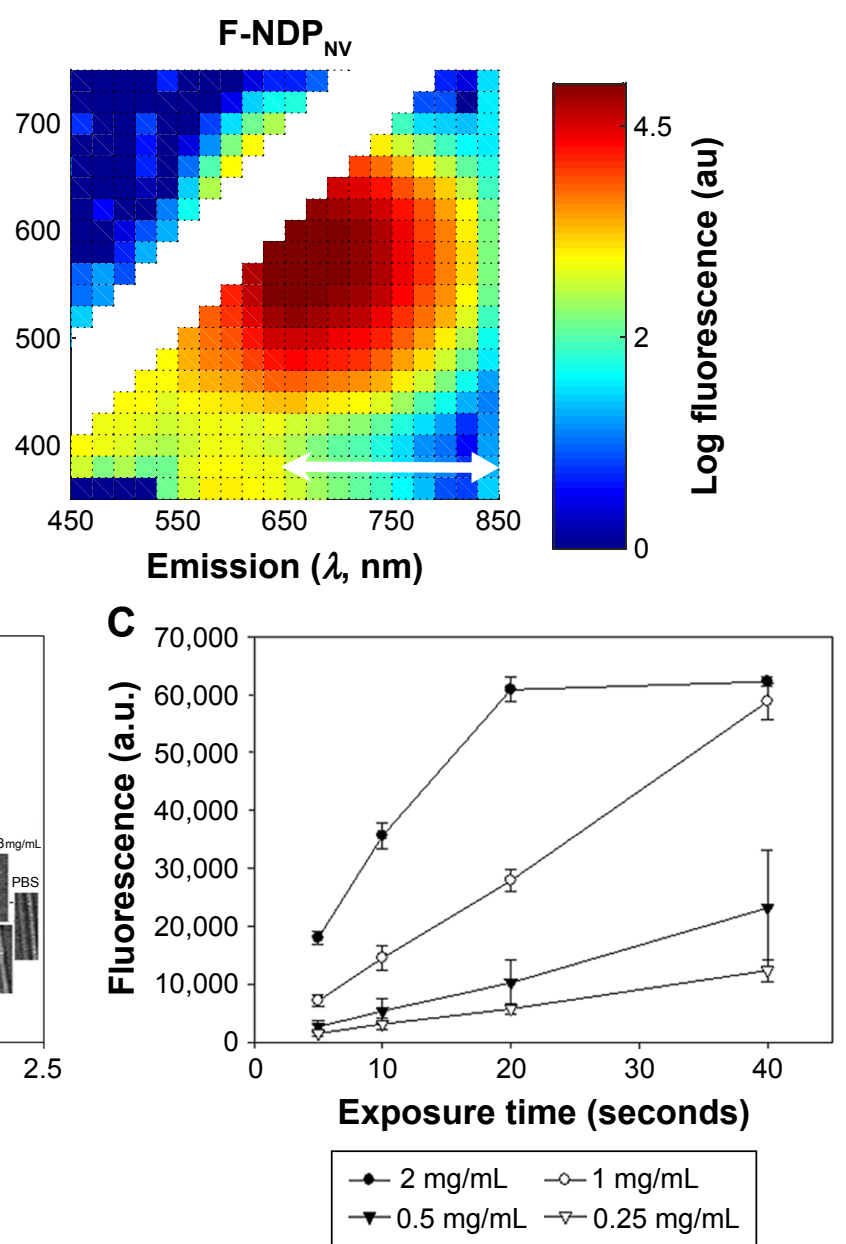

Figure I Comparison of NIR fluorescence intensity of F-NDP $\mathrm{NV}_{\mathrm{N}}$ and $\mathrm{F}-\mathrm{NDP} \mathrm{P}_{\mathrm{NVN}}$ in suspensions.

Notes: (A) F-NDP suspensions were scanned for fluorescence in a fluorescence plate reader for a range of excitations and emissions. The fluorescence was normalized by subtracting a blank well and was $\log _{10}$-processed. (B) Capillaries were filled with indicated density of F-NDP in PBS and analyzed by IVIS after 5 seconds of exposure. Insert indicates representative images of capillaries. Average fluorescence is presented in the plot. (C) Comparison of fluorescence intensity for different concentrations of $\mathrm{F}_{-\mathrm{NDP}} \mathrm{NV}_{\mathrm{NV}}$ as function of exposure time. Error bars represent SD for three to five independent experiments. $*$ Difference between F-NDP NV and F-NDP ${ }_{N V N}(p<0.0 I)$.

Abbreviations: NIR, near-infrared; F-NDP ${ }_{N V}$, F-NDP with N-V color centers; F-NDP ${ }_{\mathrm{NVN}}$, F-NDP with N-V-N color centers; F-NDP, fluorescence nanodiamond particles; $\mathrm{PBS}$, phosphate-buffered saline; IVIS, in vivo imaging system; SD, standard deviation.

PerkinElmer Inc., Akron, OH, USA) using an excitation filter set to "Cy5.5 BkG" (580-610 nm) and an emission filter set to "Cy5.5" (695-770 nm) as these filters matched best the NIR emission profile detected as described above. Imaging was completed with "binning" set to 4 , and a $10 \mathrm{~cm}$ field of view, with exposure times between 2 and 40 seconds. For imaging through biological barriers (rat and porcine skins and rat muscles), auto-fluorescence was imaged with the blue-shifted excitation "GFP" (445-490 nm) and the same emission filter "Cy5.5" (695-770 nm) with similar imaging settings as above, and subtracted from the foreground as modified from IVIS 50 protocol to compensate for the large stokes shift of the F-NDP ${ }_{\mathrm{NV}} \cdot{ }^{12}$ This correction was used for a simplified spectral un-mixing: a ratio of auto-fluorescence between the channel of interest and blue-shifted excitation channel is defined in control tissue. The same ratio is then used to subtract auto-fluorescence from the channel of interest based on the blue-shifted excitation channel in the test specimen. This operates under the assumption that the fluorophore being detected will have minimal excitation at the blue-shifted wavelength. To assess tissue penetration of NIR florescence emission from F-NDP, capillaries were placed under shaved abdominal rat skin (obtained from euthanized rats), covered with dissected rat quadriceps muscle (2-5.9 mm thick) or porcine skin (isolated from shoulder of pig obtained from a local butcher shop).

\section{Generation of carotid arterial blood clot and F-NDP infusion in rat}

Technical procedures of the $\mathrm{FeCl}_{3}$-induced vascular thrombosis model are described elsewhere. ${ }^{13,14}$ Specific modifications used in this particular work are briefly summarized here. All animal procedures were performed according to the guidelines of the US Animal Welfare Act and approved by 
the Institutional Animal Care and Use Committee at SUNY Downstate Medical Center. In brief, adult male Sprague Dawley rats (Charles River; $350 \mathrm{~g} \pm 10 \%$ body weight) were anesthetized using 4\% IF (induction, in chamber) followed by $1 \%-2 \%$ IF (maintenance) adjusted throughout the procedure. Rats were held in the supine position and subjected to surgery using clean instruments and aided by binoculars. The left carotid artery was dissected and exposed at the bifurcation region. A 5-0 surgical silk suture was wrapped below the common carotid artery (CCA), external carotid artery (ECA), and internal carotid artery (ICA). A PE-10 cannula was then inserted in the ECA for studies where F-NDP $_{\mathrm{NV}}$-Bit were injected locally. A PE-10 cannula was also inserted into the left femoral vein for studies where F-NDP $_{\mathrm{NV}}$-Bit were infused intravenously. The ICA stem was wrapped in Parafilm soaked in $50 \% \mathrm{FeCl}_{3}$ and kept in place for 10 minutes. Two to three minutes after placing the Parafilm onto the ICA, infusion of F-NDP ${ }_{\mathrm{NV}}-\mathrm{Bit}$ suspension in phosphate-buffered saline (PBS) commenced either via the $\operatorname{ECA}(\mathrm{N}=2,15 \mathrm{mg} / \mathrm{kg}$ in $1 \mathrm{~mL}$ PBS $)$ or via the femoral vein $(\mathrm{N}=6)$ at low dose $(\mathrm{N}=3,3 \mathrm{mg} / \mathrm{kg}$ in $1 \mathrm{~mL} \mathrm{PBS})$, or at high dose (N=3, $45 \mathrm{mg} / \mathrm{kg}$ in $3 \mathrm{~mL} \mathrm{PBS})$. All infusions were completed over 10 minutes. Control rats were infused with vehicle at comparable volumes and duration.

\section{Tissue fixation post-F-NDP ${ }_{N V}-$ Bit infusion}

Following the completion of particles infusion, anesthesia was augmented to produce deep hypnosis using 5\% IF. The lower aorta was quickly isolated and cut to allow blood drainage. Tissue was fixed, and residual blood removed by perfusion with $10 \mathrm{~mL}$ of $70 \%$ denatured ethanol. Dissection of both bilateral carotid artery bifurcation regions was completed after whole-body imaging (IVIS). Vessels were suspended in $70 \%$ denatured ethanol for further ex vivo NIR fluorescence evaluation.

\section{In situ and ex vivo imaging of F-NDP ${ }_{\mathrm{NV}}$ fluorescence by IVIS}

Briefly, NIR fluorescence was detected using a 580-610 nm excitation and a 695-770 $\mathrm{nm}$ emission passband with 2-second exposure, with "binning" set to 4 and a $7 \mathrm{~cm}$ field of view as described above. Auto-fluorescence was subtracted based on excitation at 445-490 $\mathrm{nm}$ under otherwise similar imaging conditions. Carotid arteries were exposed before imaging to enable clear visualization of in situ images. Following in situ imaging, carotid arterial bifurcations were removed from animals and placed on a glass plate for imaging ex vivo using identical imaging parameters to those used for in situ imaging. For each artery, the mean fluorescence intensity of the images compensated for auto-fluorescence was calculated using ImageJ (NIH, Bethesda, MD, USA).

\section{Ex vivo imaging of $F-N D P_{N V}$ by fluorescence microscopy}

Gross images of the entire carotid bifurcation region extracted from clot bearing or contralateral vessels were evaluated on a Fluoview FV1000 (Olympus, Tokyo, Japan) laser scanning confocal microscope (LSCM) using a $4 \times$ objective. NIR fluorescence emitted from $\mathrm{F}-\mathrm{NDP}_{\mathrm{NV}}$ was detected at an excitation of $543 \mathrm{~nm}$ and an emission of 655-755 nm. Confocal stacks were combined using a maximum intensity projection so that the entire vessel was brought into focus. The mean fluorescence intensity of F-NDP in each artery was calculated after subtraction of the local background using ImageJ.

\section{Isolation of F-NDP ${ }_{\mathrm{NV}}$ from vascular clot}

F-NDP were isolated from extracted carotid arteries by homogenization in RIPA lysis buffer (Teknova Inc., Hollister, CA, USA) at $100 \mathrm{mg} / \mathrm{mL}$. Aliquots $(10 \mu \mathrm{L})$ of the lysate suspension were applied on the microscope slides and analyzed under LSCM as above under 20× objective.

In animals treated with $\mathrm{F}-\mathrm{NDP}_{\mathrm{NV}}$ via the femoral artery at high dose, F-NDP ${ }_{\mathrm{NV}}$ were isolated from extracted carotid arteries by solubilizing the clot bearing vessel segment in $12 \mathrm{~N}$ hydrochloric acid (Thermo Fisher Scientific) overnight at $60^{\circ} \mathrm{C}$ at $100 \mathrm{mg} / \mathrm{mL} .{ }^{15}$ The solution was centrifuged $(14,000 \times g$ at room temperature for 10 minutes), and the pellet was washed once with distilled water. The pellet containing the insoluble $\mathrm{F}-\mathrm{NDP}_{\mathrm{NV}}-\mathrm{Bit}$ was resuspended in DI water while keeping the initial mass/volume ratio. An aliquot of the suspension was applied to a hemocytometer (Incyto Inc., Cheonan, South Korea), which was standardized for particles counting in an inverted fluorescence microscope (Olympus IX81) with $40 \times$ objective. Images of F-NDP ${ }_{\mathrm{NV}}-\mathrm{Bit}$ were taken from each observation field for counting using TRITC filter cube. Numbers of particles were calculated for the entire solubilized tissue.

\section{Statistical analysis}

Unless stated otherwise, each experiment was performed independently three times in triplicate. No outlying data were excluded. Data are represented as mean \pm standard deviation. Statistical analyses were done by the Student's $t$-test using SigmaPlot $^{\circledR} 12$ SPSS (Systat Software Inc., San Jose, CA, USA). $p<0.05$ was considered significant. 


\section{Results}

\section{Comparison of NIR fluorescence intensity of F-NDP ${ }_{\mathrm{NVN}}$ and F-NDP ${ }_{\mathrm{NV}}$}

Fluorescence profile measurements revealed NIR fluorescence in both N-V and N-V-N particles. However, fluorescence in the NIR region was 20 times greater in the N-V particles (Figure 1A). This experiment also revealed the peak excitation of $\mathrm{F}_{-\mathrm{NDP}_{\mathrm{NV}}}$ at $570 \mathrm{~nm}$ and peak emission at $670 \mathrm{~nm}$. Using this peak excitation and emission profile, NIR emission of the F-NDP was compared in the IVIS in a dose response manner (Figure $1 \mathrm{~B}$ and $\mathrm{C}$ ). Capillary studies revealed that under the same excitation conditions, NIR emission of the F-NDP ${ }_{\mathrm{NV}}$ was more effective than that of F-NDP ${ }_{\mathrm{NVN}}$ by approximately an order of magnitude (Figure 1B).

Fluorescence characteristics of F-NDP can vary with size of particle. ${ }^{16}$ The NIR emission of identical mass per milliliter of F-NDP ${ }_{\mathrm{NV}}$ was compared for particles of three different sizes (Figure 2). The lowest fluorescence intensity was observed for smallest $(100 \mathrm{~nm})$ particles, while the highest fluorescence emission was observed for $700 \mathrm{~nm}$ F-NDP. Fluorescence scaled linearly with exposure time

A
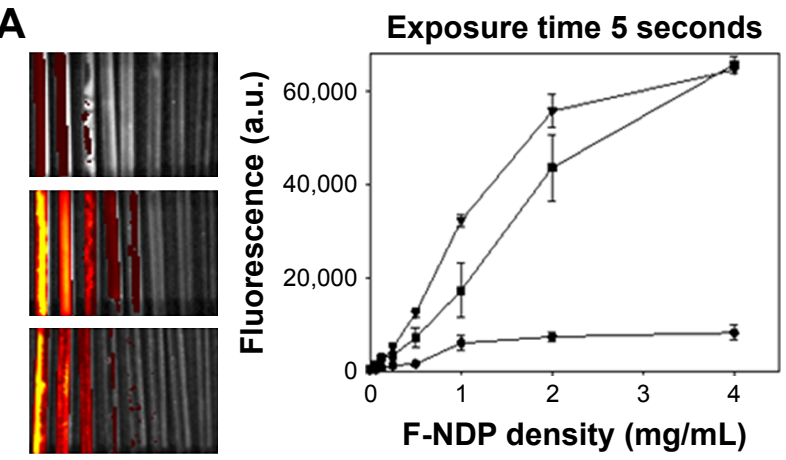

C
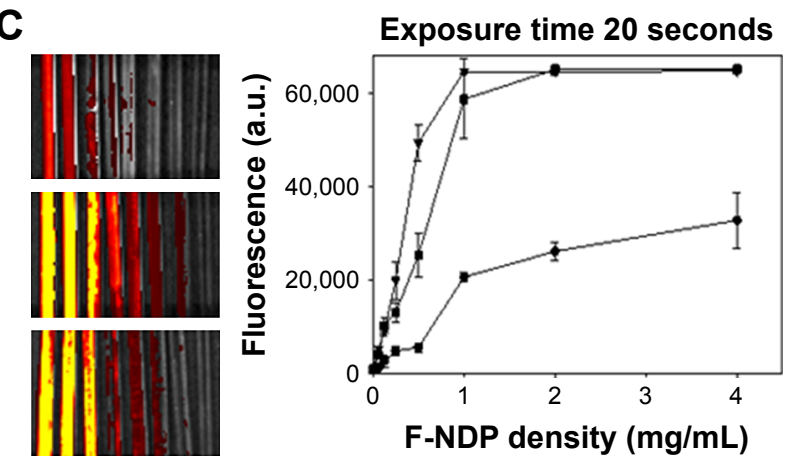

B



D

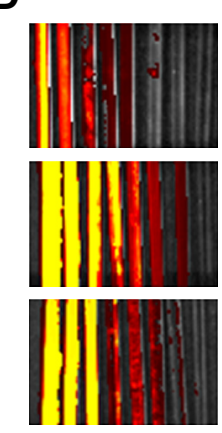

revealing no bleaching at the higher exposure times tested. While fluorescence from the $700 \mathrm{~nm}$ F-NDP ${ }_{\mathrm{NV}}$ particles saturated the detector in less than 20 seconds, longer exposure times were required to clearly display fluorescence from the F-NDP ${ }_{\mathrm{NVN}}$ particles. It is noteworthy that for the same acquisition time the NIR emission of 700 and $10,000 \mathrm{~nm}$ was not augmented. In fact, at 0.5 and $1 \mathrm{mg} / \mathrm{mL}$, the emission of the $10,000 \mathrm{~nm}$ particles was significantly lower than that of the $700 \mathrm{~nm}$ particles ( $p<0.01$, Figure $2 \mathrm{~A})$.

We then tested the ability of NIR fluorescence emitted from equivalent-sized (700 nm) F-NDP of the N-V and $\mathrm{N}-\mathrm{V}-\mathrm{N}$ strains to penetrate biological barriers as imaged in the IVIS (Figure 3). Capillaries filled with $4 \mathrm{mg} /{\mathrm{mL} \mathrm{F}-\mathrm{NDP}_{\mathrm{NV}}}$ particles could be imaged through rat skin (Figure $3 \mathrm{~A}$ ) or quadriceps muscle (Figure 3B) as well as porcine axillary vein (Figure $3 \mathrm{C}$ ) and $2.5 \mathrm{~mm}$ of defatted porcine skin (Figure 3D), while capillaries filled with F-NDP ${ }_{\mathrm{NVN}}$ particles could not be visualized with similar imaging parameters in any of these circumstances. NIR fluorescence was monitored through $2.5 \mathrm{~mm}$ of defatted porcine skin at concentrations from 1 to $4 \mathrm{mg} / \mathrm{mL}$ (30-120 $\mu \mathrm{g}$ total) (Figure 3E) as the signal did not penetrate full-thickness tissue. Porcine axillary veins 

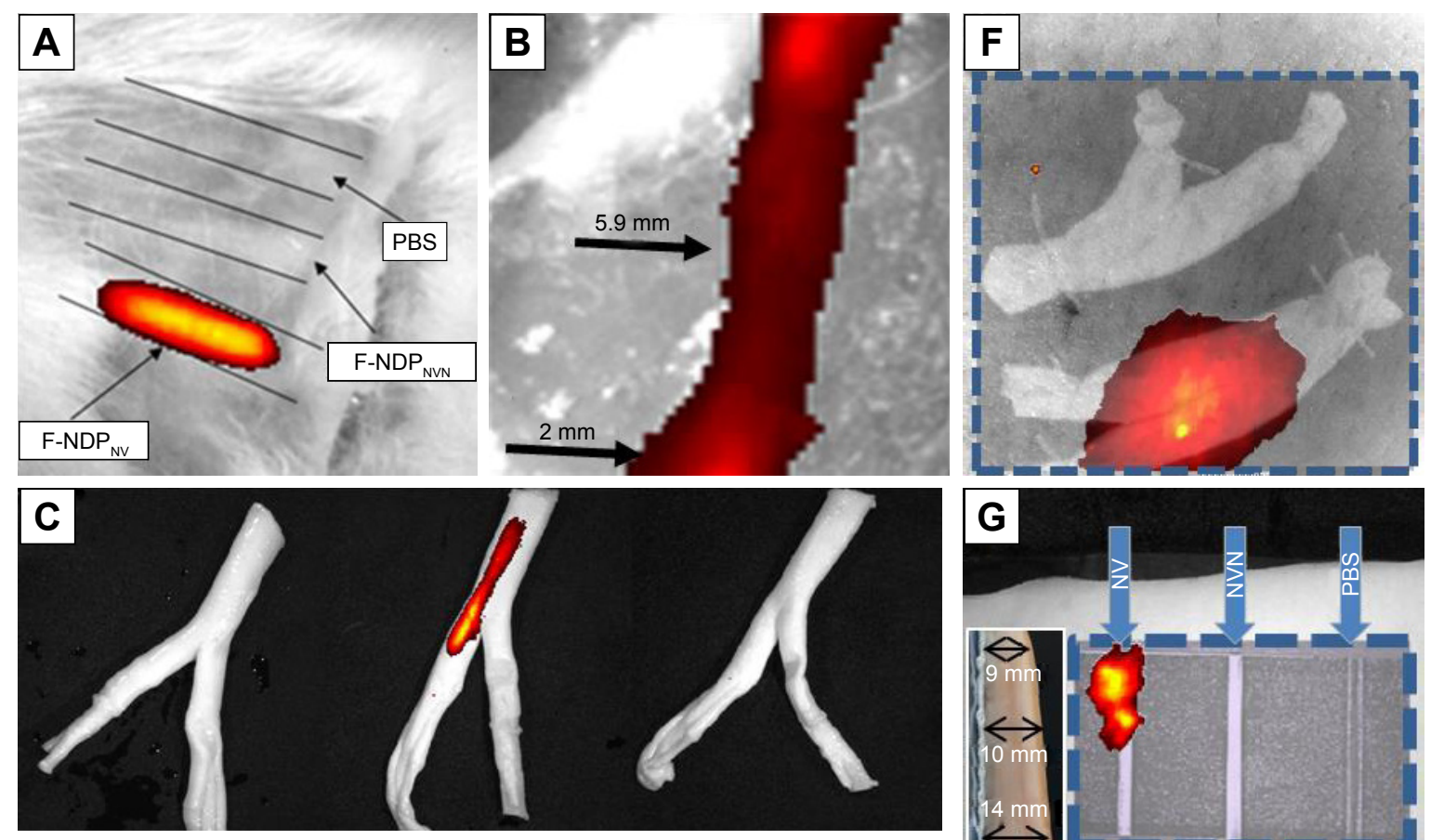

NVN

NV

PBS
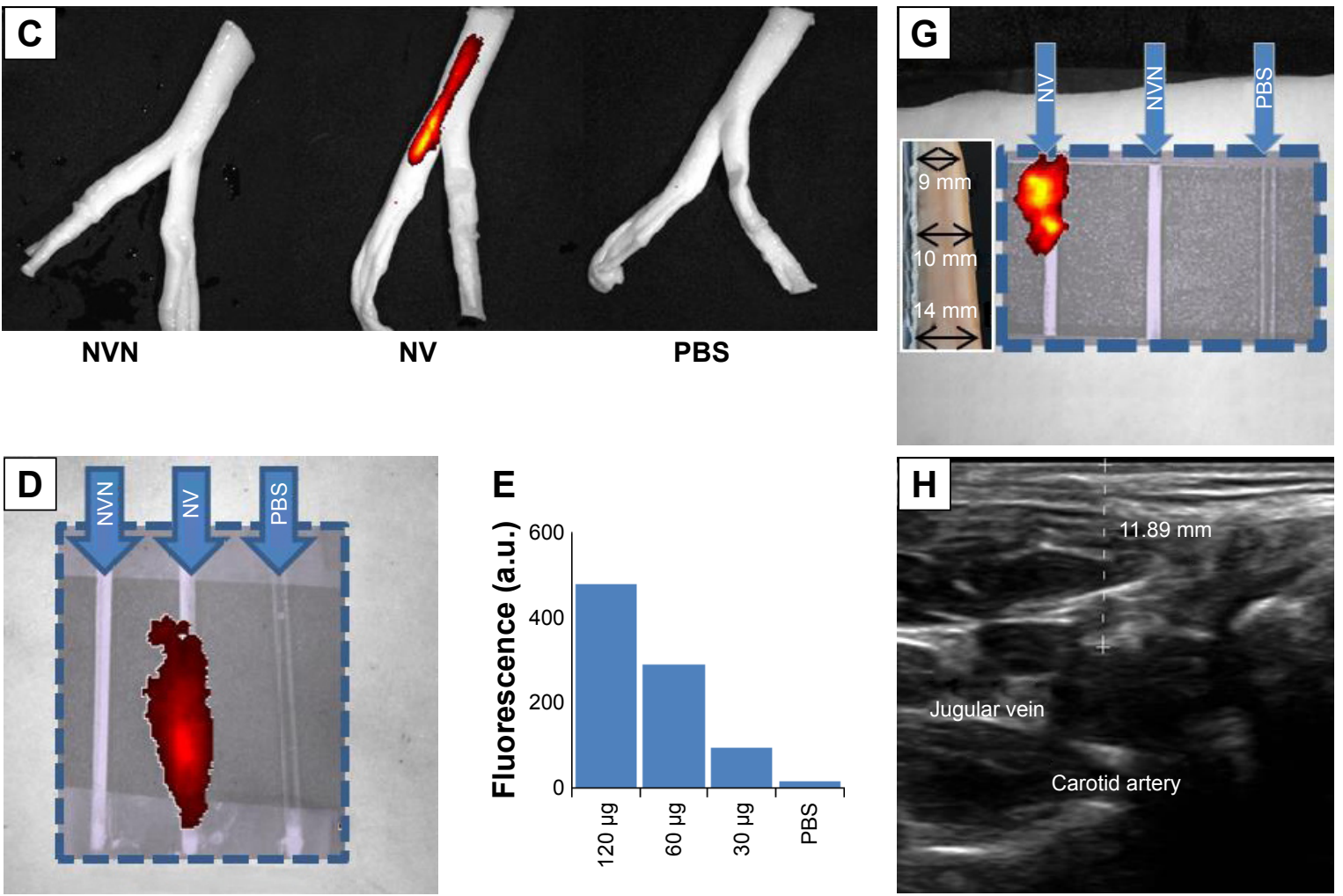

Figure 3 Comparison of the ability to detect F-NDP NIR fluorescence through different biological barriers using IVIS.

Notes: (A) Capillaries filled with F-NDP ${ }_{N V}(4 \mathrm{mg} / \mathrm{mL})$, F-NDP ${ }_{\mathrm{NVN}}(4 \mathrm{mg} / \mathrm{mL})$, or PBS were positioned under abdominal skin patch of euthanized rat. Positions of capillaries are indicated by arrows. (B) Capillary filled with F-NDP ${ }_{\mathrm{NV}}(4 \mathrm{mg} / \mathrm{mL}$ ) covered by rat quadriceps muscles ranged from $2 \mathrm{~mm}$ (flanking) to $5.9 \mathrm{~mm}$ (in the center). (C) Capillaries filled with F-NDP $(4 \mathrm{mg} / \mathrm{mL}$ ) or PBS were inserted into porcine axillary vein. (D) Capillaries filled with F-NDP (4 mg/mL) or PBS were covered with porcine skin (2.5 mm) free of subdermis. (E) Intensity of fluorescence for different concentrations of F-NDP ${ }_{\mathrm{NV}}$ through $2.5 \mathrm{~mm}$ porcine skin free of subdermis. (F) Porcine axillary veins filled with F-NDP ${ }_{\mathrm{NV}}(2 \mathrm{mg} / \mathrm{mL})$ or PBS and covered with $8 \mathrm{~mm}$ porcine skin including dermis and subdermis. (G) Capillaries filled with F-NDP $\mathrm{NV}(20 \mathrm{mg} / \mathrm{mL})$, F-NDP ${ }_{\mathrm{NVN}}(20 \mathrm{mg} / \mathrm{mL})$, and PBS (same volume) covered with porcine skin containing increased thickness of adipose tissue (presented on the insert). (H) Representative ultrasound of human carotid artery showing the artery $11.89 \mathrm{~mm}$ below the surface.

Abbreviations: F-NDP, fluorescence nanodiamond particles; NIR, near-infrared; IVIS, in vivo imaging system; F-NDP ${ }_{\mathrm{NV}}, \mathrm{F}_{-N D P}$ with N-V color centers; F-NDP ${ }_{\mathrm{NVN}}$, F-NDP with $\mathrm{N}-\mathrm{V}-\mathrm{N}$ color centers; PBS, phosphate-buffered saline.

loaded with $1 \mathrm{~mL}$ of $2 \mathrm{mg} / \mathrm{mL}$ F-NDP ${ }_{\mathrm{NV}}$ particles could be visualized through $8 \mathrm{~mm}$ of porcine skin (Figure $3 \mathrm{~F}$ ). As a final test of penetration capacity, an angled piece of fullthickness porcine skin varying from 9 to $14 \mathrm{~mm}$ in thickness was laid on top of capillaries containing $20 \mathrm{mg} / \mathrm{mL}$ ( $600 \mu \mathrm{g}$ total) of $\mathrm{F}-\mathrm{NDP}_{\mathrm{NV}}$. A detectable signal from the $\mathrm{F}-\mathrm{NDP}_{\mathrm{NV}}$ was recorded through the porcine skin up to $12 \mathrm{~mm}$ in thickness. In contrast, no light emission was detected at equal conditions of F-NDP ${ }_{\mathrm{NVN}}$ (Figure 3G). Noteworthy are data obtained by ultrasound imaging of human carotid arteries, where the carotid artery bifurcation distance from the skin surface was assessed at $14 \mathrm{~mm}$ below the skin surface (courtesy of Dr Aaron O Shiloh, unpublished data). These findings suggest a translational prospect of F-NDP ${ }_{\mathrm{NV}}$ to detect blood clot in this area if comparable particle mass can be safely deposited on a blood clot in this region (Figure 3H). 


\section{Detection of blood clot in rat model using F-NDP $\mathrm{NV}-\mathrm{Bit}$}

The results depicted in Figures 1-3 suggest that F-NDP $(700 \mathrm{~nm})$ are the preferred F-NDP strain for imaging in vivo. Therefore, all subsequent in vivo studies were carried out with F-NDP ${ }_{\mathrm{NV}}$ coupled with Bit for detection of thrombi generated in the carotid artery bifurcation of rats.

After clot formation and treatment with $\mathrm{F}-\mathrm{NDP}_{\mathrm{NV}}-\mathrm{Bit}$ via the ECA, carotid arteries were imaged in situ and removed from the animal for imaging and analysis. Injection of F-NDP via the ECA optimizes the exposure of the particles to the lesion site, thus avoiding potentially confounding variables of distribution, uptake, and elimination. Imaging of fluorescence in the IVIS scanner demonstrated strong fluorescence in situ (Figure 4A and B) in the vessel branches corresponding to the location of the clot in the exposed artery. After removing the carotid arterial bifurcations from treated and untreated animals, a strong fluorescent signal was detected (Figure 4C and $\mathrm{D}$ vs $\mathrm{E}$ and $\mathrm{F}$ ) in the $\mathrm{FeCl}_{3}$-treated arteries. This was further validated by confocal imaging confirming co-location of clot and deposited particles (Figure 4G and $\mathrm{H}$ vs I and J). As a final validation method, particles were imaged in pooled lysates (two lesions), showing large numbers of fluorescence particles in $\mathrm{F}-\mathrm{NDP}_{\mathrm{NV}}$-treated animals compared to their absence in animals treated with vehicle (Figure 4K).

Following the initial proof of feasibility of direct administration of the F-NDP ${ }_{\mathrm{NV}}$-Bit via ECA, a low $(3 \mathrm{mg} / \mathrm{kg})$ and high $(15 \mathrm{mg} / \mathrm{kg})$ dose of $\mathrm{F}-\mathrm{NDP}_{\mathrm{NV}}$-Bit was infused systemically into animals via the femoral vein. The results from animals treated with the low dose were inconclusive, as the signal was not consistently above the fluorescence level detected from carotid bifurcation isolated from control animals treated with vehicle only. Despite this, pooled lysates (three lesions) showed that F-NDP had been logged in the clots, while no particle-associated fluorescence was detectable from vehicle-treated animals (Figure 4L).

After intravenous (IV) injection of the high dose of F-NDP ${ }_{\mathrm{NV}}$-Bit, clot-associated fluorescence was demonstrated in situ in all treated animals (Figure 5A-C). Arteries with $\mathrm{FeCl}_{3}$-generated lesions and the contralateral control were then dissected and imaged independently by IVIS and confocal microscopy. A strong fluorescence signal in the treated artery was observed by both IVIS (Figure 5D-F vs $\mathrm{G}-\mathrm{I}$ ) and confocal imaging (Figure $5 \mathrm{~J}-\mathrm{L}$ vs $\mathrm{M}-\mathrm{O}$ ). The difference in brightness to that of the control artery was statistically significant (Figure $5 \mathrm{R}$ and $\mathrm{S}$ ) with $p<0.05$. Lysates collected by dissolving the carotid arteries also demonstrated a large number of fluorescent particles residing in the lesioned artery and a low, but consistent, number of particles in the contralateral control arteries (Figure 5P). This difference was highly statistically significant (Figure 5Q) with $p<0.01$.

\section{Discussion}

The two main objectives of this study were a) characterization of the two strains of the F-NDP (F-NDP ${ }_{N V}$ and F-NDP ${ }_{N V N}$ ) in terms of their NIR emission parameters and b) to identify which of the two F-NDP strains emits sufficient energy that most likely penetrates biological tissues over a distance required for the translational application in human NIR vascular pathology imaging. Given the prospect of longer (minutes) scale of imaging procedures in humans, ${ }^{8}$ an important consideration in selection of F-NDP has been the stability/durability of the NIR emission. Nanoparticles with similar robustness include quantum dots and nanorubies. Biocompatibility of many common quantum dots is questionable, ${ }^{20,21}$ though more compatible forms are under development. ${ }^{22}$ Nanorubies are an attractive though immature platform with limited commercial availability. Furthermore, the methodology to functionalize these particles is not well established compared to nanodiamonds. Other nanodiamond varieties have demonstrated satisfactory biocompatibility in vivo. ${ }^{17}$ The nanodiamonds ( $700 \mathrm{~nm}$ diameter) used in this study have not yet been studied in biological systems. However, nanoparticles in general tend to have reduced toxicity with increased diameter. ${ }^{18}$ Specifically, nanodiamonds display reduced toxicity (compared to, for example, nanotubes) with increasing diameter (tested up to $100 \mathrm{~nm}),{ }^{19}$ suggesting that the $700{\mathrm{~nm} \mathrm{~F}-\mathrm{NDP}_{\mathrm{NV}}}$ used in this study will be safe. Preliminary studies commenced at external venue suggest no mortality or morbidity in rats injected via venous port, with $700 \mathrm{~nm} \mathrm{F-NDP}{ }_{\mathrm{NV}}$ at $45 \mathrm{mg} / \mathrm{kg}$ while evaluated for weight and neurobehavioral tests for up to 5 days. Taken together, the F-NDP selected for the in vivo study reported inhere appeared to us to be a preferred choice over the other particles for this study.

Our first objective has been to systematically investigate the emission attributes each of F-NDP across four independent variables: a) the fluorescence spectrum and brightness resulting from the atomic manipulations $(\mathrm{N}-\mathrm{V}$, $\mathrm{N}-\mathrm{V}-\mathrm{N}$ ); b) particles total mass relationships to emission intensity; c) the relationship of particle diameter to emission intensity; and d) speed and extent of NIR acquisition kinetics. These variables have been exercised "head to head" between $\mathrm{N}-\mathrm{V}$ and $\mathrm{N}-\mathrm{V}-\mathrm{N}$ strains using IVIS, which we consider as well suited for such comparison due to its sensitivity and its noninvasive NIR light detection capability. ${ }^{23}$ The data presented in Figures 1 and 2 unequivocally point to the superior 



Figure $4 \mathrm{~F}-\mathrm{NDP}_{\mathrm{NV}}$-Bit infusion via external carotid artery.

Notes: Clots in the carotid arteries were imaged in situ and removed from the animal for further direct imaging and analysis. (A-F) Images of fluorescence recorded by an IVIS scanner designed for whole-animal imaging using a 580-610 nm excitation and a $695-770 \mathrm{~nm}$ emission passband with 2-second exposure. Auto-fluorescence was subtracted based on excitation at $445-490 \mathrm{~nm}$. (A and B) In situ fluorescence imaging of carotid arterial clot after treatment in duplicate animals by IVIS (separation of neck particle by dissection). Scale bar $=1 \mathrm{~cm}$. (C-F) Ex vivo fluorescence of isolated carotid artery after treatment with (C and $\mathbf{D})$ F-NDP ${ }_{\mathrm{Nv}}-\mathbf{B i t}_{\mathrm{t}}$ or (E and $\left.\mathbf{F}\right)$ vehicle control of saline. Scale bar $=\mathrm{I} \mathrm{mm}$. (G-J) Confocal image stacks were taken on Olympus FV 1000. F-NDP $\mathrm{Nv}^{-}$-Bit fluorescence was detected at an excitation of $543 \mathrm{~nm}$ and an emission of 655-755 nm. Background fluorescence was collected from the same excitation, with emissions of 555-625 nm, and was subtracted from the foreground. Scale bar =I mm.


with untreated contralateral artery. (L-N) Clots were dissolved in RIPA lysis buffer, and replicates were combined together to form a lysate. Aliquots of the lysate were then

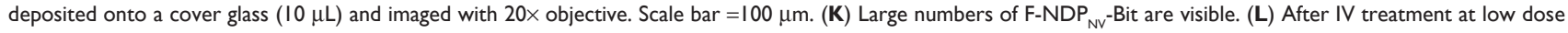
(I mg), F-NDP ${ }_{\mathrm{Nv}}$-Bit were found in the lysate at the site of clot formation. (N) Almost no fluorescent particles are detected in saline control.

Abbreviations: F-NDP ${ }_{\mathrm{NV}}$-Bit, fluorescence nanodiamond particles with N-V color centers coupled to bitistatin; IVIS, in vivo imaging system; IV, intravenous; LICA, left internal carotid artery; LECA, left external carotid artery; LCCA, left common carotid artery; RECA, right external carotid artery; RICA, right internal carotid artery; RCCA, right common carotid artery; PFA, paraformaldehyde.

prospect of the F-NDP ${ }_{\mathrm{NV}}$ strain to meet best in vivo NIR fluorescent penetration through biological tissues.

Our data demonstrating superior NIR fluorescence of F-NDP $_{\mathrm{NV}}$ vs F-NDP ${ }_{\mathrm{NVN}}$ are in line with prior findings comparing the same color centers in diamonds. ${ }^{24}$ Furthermore, the effect of nanodiamond size on fluorescent characteristics of commercially available nanodiamond particles has been demonstrated in other size ranges and diamond types. ${ }^{16,25}$ Specifically, in our study, the $700{\mathrm{~nm} \mathrm{~F}-\mathrm{NDP}_{\mathrm{NV}}}$ exhibited 10-60 times higher NIR emission intensity than the 


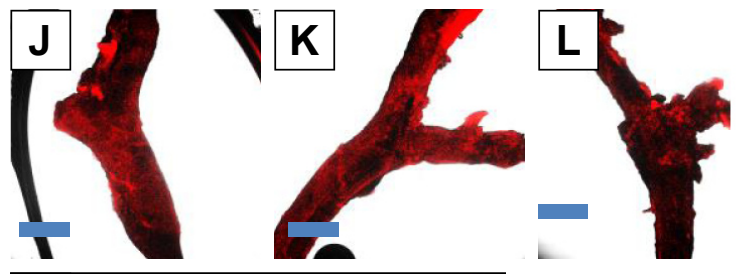

Q

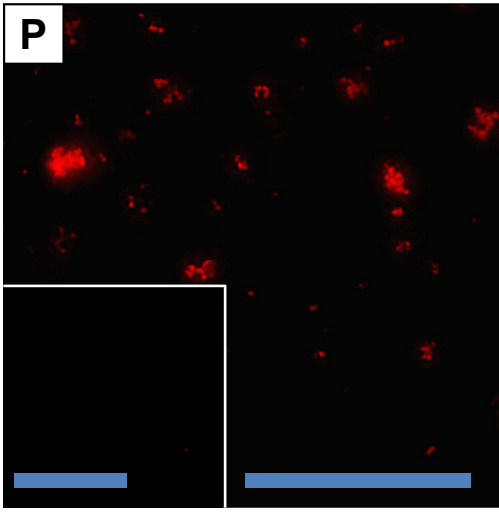

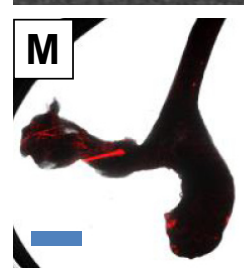

$\mathbf{R}$

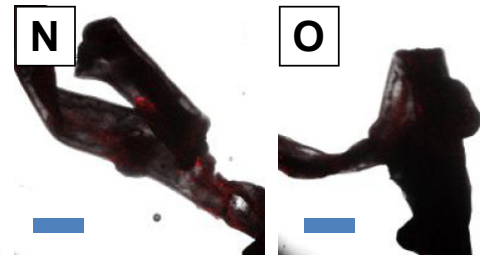

S


Figure 5 F-NDP ${ }_{\mathrm{NV}}$-Bit intravenous infusion.

Notes: (A-J) Images of fluorescence captured using an IVIS scanner designed for whole-animal imaging using a 580-610 nm excitation and a $695-770 \mathrm{~nm}$ emission passband with 2-second exposure. Auto-fluorescence was subtracted based on excitation at $445-490 \mathrm{~nm}$. (A-C) Gross image indicating fluorescence of carotid arterial clot after treatment in triplicate animals is visible via IVIS after exposure of artery. Scale bar $=I \mathrm{~cm}$. (D-I) Ex vivo fluorescence of carotid artery after (D-F) $F-N D P_{N V}-B_{i t}$ treatment

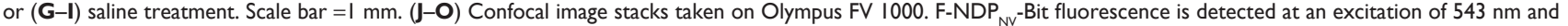
an emission of $655-755 \mathrm{~nm}$. Scale bar $=1 \mathrm{~mm}$. Fluorescence of carotid artery after (J-L) F-NDP Nv $^{-B i t}$ treatment or (M-O) saline treatment. (P) Lysates from solubilized carotid arteries were imaged, and F-NDP ${ }_{N v}$-Bit were counted by hemocytometer. Contralateral untreated control is presented in insert. Scale bar $=100 \mu m$. $(\mathbf{Q})$ Total number of F-NDP ${ }_{\mathrm{NV}}$-Bit detected per carotid bifurcation in the clotted side compared with the contralateral control as counted by hemocytometer. (R and $\left.\mathbf{S}\right)$ Brightness after subtraction of background via IVIS (D-I) and LSCM (J-O) imaging of treated and contralateral untreated carotid bifurcations. Error bars represent standard deviation. $* p<0.05$ and ${ }^{* *} p<0.01$ vs control by $t$-test.

Abbreviations: $\mathrm{F}_{-N D P_{N V}}-$ Bit, fluorescence nanodiamond particles with N-V color centers coupled to bitistatin; IVIS, in vivo imaging system; LSCM, laser scanning confocal microscope; F-NDP-Bit, fluorescence nanodiamond particles coupled to bitistatin.

F-NDP $_{\mathrm{NVN}}$. Of note, NIR emission from F-NDP ${ }_{\mathrm{NV}}$ was not directly correlated with particle size, and was maximized for $700 \mathrm{~nm} \mathrm{F-NDP}{ }_{\mathrm{NV}}$. At constant particle mass loading, the $700 \mathrm{~nm}$ particles were approximately four times brighter in IVIS images, and consistently and across all acquisition periods tested; NIR fluorescence emission from 10,000 nm particles was weaker than from $700 \mathrm{~nm}$ particles (Figure 2).
While 10,000 nm F-NDP ${ }_{\mathrm{NV}}$ are not likely to be useable for IV injection, the $10,000 \mathrm{~nm}$ particles demonstrated that under equal mass conditions particle brightness was not correlated with diameter, but may be maximized for particles of a specific diameter range. This observation suggests that the "best" particles available will likely be within a size range suitable for IV injection and is the reason we used the $700 \mathrm{~nm}$ 
for further experiments. A careful physicochemical analysis of the possible origins of the size-dependent fluorescence is beyond the scope of this study. Here, we focused on surveying commercially available nanodiamonds to select the best candidates for further testing in our system. Certain applications of NIR technology for in vivo diagnostics (eg, pharmacokinetic and organ distribution restrictions) could require more restricted size and generally favor maximum brightness at minimum size.

Commensurate with the physical properties delineated for the F-NDP ${ }_{\mathrm{NV}}$ (Figures 1 and 2), the data in Figure 3 further support the potential of the F-NDP ${ }_{\mathrm{NV}}$ to support in vivo vascular clot imaging. Figure 3 presents several conditions where tissues penetration of NIR fluorescence emitted from F-NDP $_{\mathrm{NV}}$ was tested. The maximum distance detectable by NIR through porcine skin, used as a human skin analog (Figure $3 \mathrm{G}$ ), was $12 \mathrm{~mm} .{ }^{26}$ Figure $3 \mathrm{H}$ presents an ultrasound recording of normal human carotid artery bifurcation annotated for the ICA, ECA, and CCA. The linear dashed bar in Figure $3 \mathrm{H}$ indicates $11.89 \mathrm{~mm}$ depth of the human carotid artery bifurcation from the vertical distance from the neck surface (courtesy of Dr Aaron O Shiloh). Considering the dense epidermis of porcine shoulder skin compared to human neck and the distance of the IVIS camera from the target to be monitored vs the use of a similar hand-held device placed directly on the skin, we posit that NIR fluorescence imaging of a clot in the carotid artery bifurcation is likely to be within an achievable diagnostic opportunity. Furthermore, NIR fluorescence recorded in Figure $3 \mathrm{H}$ represents a source generated from $\sim 670 \mu \mathrm{g}$ of $700 \mathrm{~nm} \mathrm{F-NDP}{ }_{\mathrm{NV}}$ particles. The same amount of particles tagged onto human clot in this region could enable clot detection by NIR fluorescence imaging. Results in Figure 5Q indicate a minimum of $0.7 \%$ of injected dose was captured in the lesion of interest $\left(2 \times 10^{8}\right.$ particles/mg in dose), which would imply a required dose of approximately $100 \mathrm{mg}(1.4 \mathrm{mg} / \mathrm{kg})$ to reach a similar emission profile in human. In preliminary studies in rat, doses as high as $45 \mathrm{mg} / \mathrm{kg}$ were well tolerated for 5 days without any adverse events recorded (data not shown).

Recent advances in nanodiamond imaging suggest the possibility to increase the signal-to-noise ratio and sensitivity over an order of magnitude over conventional detection methods. ${ }^{27}$ Therefore, the information collected in our in vitro and ex vivo experiments summarized in Figures 1-3 provided a compelling case for initiation of in vivo studies in support for our second objective.

The in vivo studies were performed with $\mathrm{F}-\mathrm{NDP}_{\mathrm{NV}}-\mathrm{Bit}$ covalently coupled with bitistatin. The procedure calls for EDC-mediated covalent heterobifunctional coupling, yielding a stable amide bond, which is resilient in biological systems. ${ }^{28}$ These F-NDP ${ }_{\mathrm{NV}}$-Bit were administered systemically to anesthetized rats subjected to an established carotid artery clot procedure at the site of the bifurcation. In our pilot translational study, F-NDP ${ }_{\mathrm{NV}}$-Bit were first administered via the ECA ("high dose", $15 \mathrm{mg} / \mathrm{kg}$ ) or via the femoral artery ("low dose", $3 \mathrm{mg} / \mathrm{kg}$ ). This experimental design was selected since pharmacokinetics and particles distribution dynamics are as yet unknown. Therefore, to avoid possible loss of significant amount of particles via "first-path" elimination or tissue distribution (potential "false-negative" outcome), injection via the ECA ascertains maximum exposure of the particles to the clot in the targeted region. To assess and confirm specific co-localization of F-NDP ${ }_{\mathrm{NV}}$-Bit in the blood, three independent methods were deployed: a) IVIS total body imaging (Figure 4A and B); b) LSCM analysis of extracted vessels carrying clots (Figure 4C and D); and c) direct count of particles extracted from clot-bearing vessels after solubilization of all organic materials (Figure 4L). Association of F-NDP ${ }_{\mathrm{NV}}$ by infusion of a high dose of F-NDP ${ }_{\mathrm{NV}}-\mathrm{Bit}$ via the ECA is easily detected by all three methods. However, infusion of a low dose $(3 \mathrm{mg} / \mathrm{kg})$ of $\mathrm{F}-\mathrm{NDP}_{\mathrm{NV}}-\mathrm{Bit}$ via the femoral vein failed to detect emission by either IVIS or LSCM (Figure 4E and F). A low amount of particles was counted in the clot extract (Figure 4M), yet it is not clear whether this very small number is specifically clot associated or loaded into the vessel wall via "vasa-vasorum" or both. It is clear however that the low dose of F-NDP ${ }_{\mathrm{NV}}$-Bit could not produce a credible emission signal that could be detected by IVIS or LSCM, even if particles were in fact target to the clot.

Since administration F-NDP ${ }_{\mathrm{NV}}$-Bit via the ECA is not a procedure of potential translation to the diagnosis of human vascular clots, we intravenously administered F-NDP $\mathrm{NV}^{-}$ Bit at a higher dose $(15 \mathrm{mg} / \mathrm{kg}, \mathrm{N}=3)$. As illustrated in Figure $5 \mathrm{~A}-\mathrm{C}$, all three animals tested displayed a strong fluorescence signal emanating from the carotid bifurcation zone (IVIS), as also clearly visible in the fluorescence of isolated vessels (Figure 5D-I) and under inspection by LSCM (Figure 5J-O). Particles were also present in large numbers in solubilized clot-bearing vessels (Figure 5P). Figure 5Q-S shows the quantitative analysis of the robust deposition of particles in clot-bearing vessels vs the contralateral control.

In summary in this work, we extended previous observations regarding the superior NIR fluorescence of F-NDP ${ }_{\mathrm{NV}}$ over F-NDP ${ }_{\mathrm{NVN}}$ and demonstrated a corresponding ability for enhanced depth of imaging through biological tissue. 
Furthermore, we provided evidence that the F-NDP ${ }_{\mathrm{NV}}$ deployed in this study can associate with clot in vivo, such as a thrombus formed in a rat carotid artery bifurcation model. The proof of concept here is based on three independent measures or the NIR fluorescence detected at the in situ clot formation, including direct counting of fluorescence particles isolated from the extracted clot. Our data demonstrate the possibility to detect by imaging the NIR fluorescence emitted from F-NDP ${ }_{\mathrm{NV}}$ over a distance corresponding to that present in vascular pathology (eg, clot in the carotid artery bifurcation). If successfully translated to clinical practice, this minimally invasive procedure, conducted in ambulatory settings, could enhance preventative measures, such as earlier initiation of anti-thromboembolic medications.

\section{Acknowledgments}

This study was supported in part by Debina Diagnostic Inc. Additional support was provided by a grant from the Moulder Center for Drug Discovery (CM and PIL) and a Research Bridge Funding Award (PIL) from the Office of the Vice President for Research Administration (OVPR).

\section{Disclosure}

The authors report no conflicts of interest in this work.

\section{References}

1. Sidney S, Quesenberry CP Jr, Jaffe MG, et al. Recent trends in cardiovascular mortality in the United States and public health goals. JAMA Cardiol. 2016;1(5):594-599.

2. Guirguis-Blake JM, Evans CV, Senger CA, O'Connor EA, Whitlock EP. Aspirin for the primary prevention of cardiovascular events: a systematic evidence review for the U.S. preventive services task force. Ann Intern Med. 2016;164(12):804-813.

3. Skovronsky D, Feuerstein G. Imaging biomarkers for diagnosis, prognosis and treatment of Alzheimer's disease. In: Rudolph AS, editor. Alzheimer's Disease Targets for New Clinical Diagnostic and Therapeutic Strategies. Boca Raton, FL: CRC Press; 2012:103-116.

4. Marcinkiewicz C, Gersetnhaber JA, Sternberg M, Lelkes PI, Feuerstein G. Bitistatin-functionalized fluorescent nanodiamond particles specifically bind to purified human platelet integrin receptor $\alpha \operatorname{IIb} \beta 3$ and activated platelets. Int J Nanomedicine. 2017;12:3711-3720.

5. Purtov KV, Petunin AI, Burov AE, Puzyr AP, Bondar VS. Nanodiamonds as carriers for address delivery of biologically active substances. Nanoscale Res Lett. 2010;5(3):631-636.

6. Gismondi A, Nanni V, Reina G, Orlanducci S, Terranova ML, Canini A. Nanodiamonds coupled with 5,7-dimethoxycoumarin, a plant bioactive metabolite, interfere with the mitotic process in B16F10 cells altering the actin organization. Int $J$ Nanomedicine. 2016;11:557-574.

7. Liu Y, Chen S, Quan X, et al. Boron and nitrogen codoped nanodiamond as an efficient metal-free catalyst for oxygen reduction reaction. J Phys Chem C. 2013;117(29):14992-14998.

8. Reineck P, Francis A, Orth A, et al. Brightness and photostability of emerging red and near-IR fluorescent nanomaterials for bioimaging. Adv Opt Mater. 2016;4(10):1549-1557.
9. Igarashi R, Yoshinari Y, Yokota H, et al. Real-time background-free selective imaging of fluorescent nanodiamonds in vivo. Nano Lett. 2012; 12(11):5726-5732.

10. Hara T, Bhayana B, Thompson B, et al. Molecular imaging of fibrin deposition in deep vein thrombosis using fibrin-targeted near-infrared fluorescence. JACC Cardiovasc Imaging. 2012;5(6):607-615.

11. Grabarek Z, Gergely J. Zero-length crosslinking procedure with the use of active esters. Anal Biochem. 1990;185(1):131-135.

12. Troy T, Jekic-McMullen D, Sambucetti L, Rice B. Quantitative comparison of the sensitivity of detection of fluorescent and bioluminescent reporters in animal models. Mol Imaging. 2004;3(1):9-23.

13. Feuerstein GZ, Toomey JR, Valocik R, Koster P, Patel A, Blackburn MN. An inhibitory anti-factor IX antibody effectively reduces thrombus formation in a rat model of venous thrombosis. Thromb Haemost. 1999; 82(5):1443-1445.

14. Toomey JR, Blackburn MN, Storer BL, Valocik RE, Koster PF, Feuerstein GZ. Comparing the antithrombotic efficacy of a humanized anti-factor IX (a) monoclonal antibody (SB 249417) to the low molecular weight heparin enoxaparin in a rat model of arterial thrombosis. Thromb Res. 2000;100(1):73-79.

15. Daulton TL, Kirk MA, Lewis RS, Rehn LE. Production of nanodiamonds by high-energy ion irradiation of graphite at room temperature. Nucl Instrum Methods B. 2001;175-177:12-20.

16. Chung PH, Perevedentseva E, Cheng CL. The particle size-dependent photoluminescence of nanodiamonds. Surf Sci. 2007;601(18):3866-3870.

17. Tsai LW, Lin YC, Perevedentseva E, Lugovtsov A, Priezzhev A, Cheng CL. Nanodiamonds for medical applications: interaction with blood in vitro and in vivo. Int J Mol Sci. 2016;17(7):1111.

18. Handa T, Hirai T, Izumi N, et al. Identifying a size-specific hazard of silica nanoparticles after intravenous administration and its relationship to the other hazards that have negative correlations with the particle size in mice. Nanotechnology. 2017;28(13):135101.

19. Liu KK, Cheng CL, Chang CC, Chao JI. Biocompatible and detectable carboxylated nanodiamond on human cell. Nanotechnology. 2007; 18(32):325102-325111.

20. Liu F, Ye W, Wang J, Song F, Cheng Y, Zhang B. Parallel comparative studies on toxicity of quantum dots synthesized and surface engineered with different methods in vitro and in vivo. Int J Nanomedicine. 2017;12: $5135-5148$.

21. Derfus AM, Chan WCW, Bhatia SN. Probing the cytotoxicity of semiconductor quantum dots. Nano Lett. 2003;4(1):11-18.

22. Sakai $Y$, Huang H, Hanada S, Niino T. Toward engineering of vascularized three-dimensional liver tissue equivalents possessing a clinically significant mass. Biochem Eng J. 2010;48(3):348-361.

23. Leblond F, Davis SC, Valdés PA, Pogue BW. Pre-clinical whole-body fluorescence imaging: review of instruments, methods and applications. J Photochem Photobiol B. 2010;98(1):77-94.

24. Rand SC. Synthetic Diamond for Color Center Lasers. Berlin: Springer; 1986.

25. Wee TL, Mau YW, Fang CY, Hsu HL, Han CC, Chang HC. Preparation and characterization of green fluorescent nanodiamonds for biological applications. Diam Relat Mater. 2009;18(2-3):567-573.

26. Summerfield A, Meurens F, Ricklin ME. The immunology of the porcine skin and its value as a model for human skin. Mol Immunol. 2015;66(1):14-21.

27. Sarkar SK, Bumb A, Wu X, et al. Wide-field in vivo background free imaging by selective magnetic modulation of nanodiamond fluorescence. Biomed Opt Express. 2014;5(4):1190-1202.

28. Patri AK, Kukowska-Latallo JF, Baker JR Jr. Targeted drug delivery with dendrimers: comparison of the release kinetics of covalently conjugated drug and non-covalent drug inclusion complex. Adv Drug Deliv Rev. 2005;57(15):2203-2214. 
International Journal of Nanomedicine

Dovepress

\section{Publish your work in this journal}

The International Journal of Nanomedicine is an international, peerreviewed journal focusing on the application of nanotechnology in diagnostics, therapeutics, and drug delivery systems throughout the biomedical field. This journal is indexed on PubMed Central, MedLine, CAS, SciSearch $\AA$, Current Contents $\AA /$ Clinical Medicine,

Journal Citation Reports/Science Edition, EMBase, Scopus and the Elsevier Bibliographic databases. The manuscript management system is completely online and includes a very quick and fair peer-review system, which is all easy to use. Visit http://www.dovepress.com/ testimonials.php to read real quotes from published authors.

Submit your manuscript here: http://www.dovepress.com/international-journal-of-nanomedicine-journal 